\title{
Predicted Decoupling for Coexistence Between WiFi and LTE in Unlicensed Band
}

\author{
Feng Tian, Member, IEEE, Yue Yu, Xu Yuan, Member, IEEE, Bin Lyu, Member, IEEE, \\ and Guan Gui, Senior Member, IEEE
}

\begin{abstract}
Wireless fidelity (WiFi) and long term evolution (LTE) coexistence system has attracted significant attention recently. However, most studies on coexistence only consider the issues about downlink performance and fairness between WiFi network and LTE network. In this paper, we take a comprehensive consideration of both the performance of WiFi and LTE, to propose a downlink/uplink decoupling (DUDe) scheme for the fairness of the coexistence system. Through the process of modeling construction, problem formulation with reformulation, solution and simulations, we illustrate that DUDe can achieve proportional fairness in terms of sum throughput and load balancing between uplink and downlink. In our decoupling model for coexistence between WiFi and LTE, resource allocation is under adaptive spectrum partitioning and users can access each network of WiFi and LTE. Moreover, to make our proposal more realistic, we adopt support vector machine (SVM) to predict the total number of users in WiFi and LTE coexistence system, where the predictive simulation shows its high prediction accuracy with low complexity. We further apply the prediction results to the optimization problem of DUDe. Simulation results manifest that the proposed DUDe scheme has better performance in terms of sum throughput. The sum throughput of DUDe scheme can come up to almost twice more than that of coupled association scheme. The result shows that DUDe can also achieve load balance between uplink and downlink in the coexistence system.
\end{abstract}

Index Terms-Downlink/uplink decoupling, proportional fairness, support vector machine, coexistence system.

\section{INTRODUCTION}

Owning to the rapid expansion of mobile Internet and the widespread use of smart terminal devices such as mobile

Manuscript received XXX, XX, 2019; revised XXX, XX, 2019.

This work was supported in part by the National Natural Science Foundation of China under Grants 61772287, 61671252 and 61772286, in part by the Key University of Science Research Project of Jiangsu Province under Grant 18KJA510004, in part by the project of Jiangsu High Technology Research Key Laboratory for Wireless Sensor Networks under Grant WSNLBKF 201802, in part by the "333" project of Jiangsu Province under Grant BRA2017401, in part by the Natural Science Foundation of Jiangsu Province under Grant BK20161518, and in part by the open research fund of National Mobile Communications Research Laboratory, Southeast University under Grant 2018D05, in part by Project Funded by the National Science and Technology Major Project of the Ministry of Science and Technology of China under Grant TC190A3WZ-2, the Jiangsu Specially Appointed Professor under Grant RK002STP16001, the Innovation and Entrepreneurship of Jiangsu High-level Talent under Grant CZ0010617002, the Summit of the Six Top Talents Program of Jiangsu under Grant XYDXX-010, the 1311 Talent Plan of Nanjing University of Posts and Telecommunications. (Corresponding author: Guan Gui)

F. Tian, Y. Yu, B. Lyu, and G. Gui are with the Key Laboratory of Broadband Wireless Communication and Sensor Network Technology, Ministry of Education, Nanjing University of Posts and Telecommunications, Nanjing 210003, China. (E-mails: \{tianf, 1016010406, blyu, guiguan $\} @$ njupt.edu.cn)

X. Yuan is with University of Louisiana at Lafayette, Lafayette, LA 70504 USA. (E-mail: yuanxuyx @gmail.com)
TABLE I: Adv. and disad. of existing coexistence between WiFi and LTE.

\begin{tabular}{|l|l|l|}
\hline Method & Advantage & Disadvantage \\
\hline Experiment only [17] & Accurate & No derivation \\
\hline Human central [18] & Semi-adaptive & Reduce satisfaction \\
\hline Adaptive access [19] & More access & Complex \\
\hline LBT [20][21] & Graceful coexistence & Feasible or not \\
\hline Duty circle [22] & WiFi improving & Losing time \\
\hline Cognitive coexist [23] & Near-optimal access & Complex \\
\hline Couple [24] & Optimal QoS & Unfairness \\
\hline Decouple[25]-[27] & Fairness & No prediction \\
\hline
\end{tabular}

phones, laptops and others, the demand of mobile data traffic in cellular systems like long term evolution (LTE) is increasing exponentially [1]-[8]. From 2010 till now, mobile data traffic raises up to one thousand times [9]. But the spectrum resources for wireless communications are valuable and limited while the demand for higher transmission rates increases incredibly. Therefore, how to further improve the capacity of existing communication systems becomes a key issue in the communication field.

However, there are a large quantity of spectrum resources in the unlicensed band, which are currently utilized by other wireless systems like wireless fidelity (WiFi). For example, in $5 \mathrm{GHz}$ band, there is up to $500 \mathrm{MHz}$ of spectrum bandwidth available. In order to overcome spectrum shortage, we plan to apply LTE in the unlicensed spectrum, known as LTE$\mathrm{U}$ [10], [11], which is deemed as a promising solution to support the ultra-capacity foreseen in a few years. According to the report of Qualcomm and Huawei [12], [13], LTE-U shows a lot of advantages of carrying more mobile data traffic and bringing users higher quality of mobile communication experiences. However, the rapid development of LTE-U also raises concern about whether or not LTE-U can coexist with WiFi harmoniously and fairly. Typically, for channel access, an LTE system always adopts a fixed time-frequency structure while a WiFi system uses a mechanism based on contention [14]-[16]. Due to the different access mechanisms between those two systems, the coexistence in the same unlicensed band may lead to some problems, such as unfairness between WiFi and LTE, and between uplink and downlink.

There have been many existing studies on investigating coexistence mechanisms for WiFi and LTE to obtain optimal network performance [17]-[27]. The advantage and disadvantage of them can be found in Table I. In [19], J. Xiao et al. proposed an optimal mechanism of channel access by providing LTE and WiFi users with equal access opportunities. In [20], [21], they used listen-before-talk (LBT) mechanism for detection of channel occupation and collision avoidance between different 
networks. In [22], E. Almeida et. al introduced the dutycircle mechanism, using almost blank sub-frames technique to enable LTE mute for a certain ratio of time slot, where WiFi transmission can be guaranteed first. And how to design the ratio of duty circle to optimize the throughput was dicussed in [23]. Moreover, since the real-time applications of mobile networks are continuously improved, the traffic in uplink has greatly increased than before. Therefore, the fairness between uplink and downlink becomes more important. However, most existing studies only focus on the analysis of downlink, so that a full consideration of both uplink and downlink should be emphasized. In heterogeneous networks, coupled association may result in unfairness between uplink and downlink [24]. Correspondingly, the basic idea of downlink/uplink decoupling (DUDe) has been given in [25]-[27]. DUDe can balance the data rates of uplink and downlink, which has already been sustained by the current LTE/LTE-A specifications. Because of the possibility that DUDe can be carried out for the case of various base stations (BSs) in cellular networks, it's important and necessary for LTE-U to implement DUDe. In this paper, we consider the coexistence between an LTE-U network and a WiFi network as shown in Fig. 1. And we propose a decoupling scheme, i.e., DUDe, to enable users to optimally decide which network to access their uplinks and downlinks. This scheme may achieve the goal of improving the performance of the whole coexistence system and realize the proportional fairness in terms of sum throughput between uplink and downlink. At the same time, an adaptive resource allocation framework in the unlicensed band will be given to guarantee the requirements of fairness.

Besides, to make our proposal more realistic, we will consider machine learning algorithm based on the regression model for predicting the total number of users in coexistence system between WiFi and LTE. Then the predicted results can be used in the formulation of the above fairness problem with DUDe. Due to high prediction accuracy with low complexity, support vector machine (SVM) can be used to extensively predict system characteristics in the future [28]. In this paper, hence, we will apply SVM to predict the total number of users. Under this case, based on the prediction and further analysis of associations of users' behaviors, we can not only allocate appropriate spectrum resources for the coexistence system but also obtain load balance between uplink and downlink in the future.

The main contribution of this paper is to develop a novel, adaptive spectrum partitioning to optimize the sum throughput and obtain load balance with DUDe in the coexistence system between WiFi and LTE. Different from traditional wireless communication systems, our proposed method enables users to optimally choose their uplinks and downlinks and access different networks. This scheme can take full advantage of spectrum resources and achieve fairness between uplink and downlink of the coexistence system. We also adopt adaptive spectrum partitioning method to allow users to choose their optimal resource allocation. Besides, we apply the SVM algorithm to predict the total number of users in the coexistence network. According to the predicted users' number by SVM, we can allocate an appropriate quantity of spectrum resources for the coexistence system. And we can further analyze the associations of users' behavior. Also we can achieve load balance between uplinks and downlinks in the future. Therefore, our proposed DUDe scheme based on SVM prediction can be used for analyzing not only static problems but also dynamic problems of the coexistence network.

The remainder of this paper is organized as follows. In Section II, the feasibility and benefits of applying DuDe scheme are introduced in WiFi and LTE coexistence system. In Section III, we give a mathematic model and its formulation for coexistence between WiFi and LTE. In Section IV, we use SVM to predict the users' number in the coexistence network. In Section V, numerical simulation results are presented. And Section VI concludes this paper.

\section{Feasibility and Benefits}

In this section, we give a brief introduction of the feasibility of DUDe in the coexistence system, and some discussions on DUDe under the base of theoretical and practical perceptions.

The conventional mobile networks such as the $2 \mathrm{G}-4 \mathrm{G}$ are always designed to couple the uplink and downlink. In other words, mobile users should tie with the same BS or access point (AP) in both uplink and downlink. This method is sufficient for the homogeneous networks due to the similar or same power level transmitted by all the BSs. Meanwhile, the homogeneous network is designed mainly based on the downlink, because the throughput requirement of the downlink is often higher than that of the uplink in traditional networks. The associations in homogeneous networks only take into account the received signal power [27]. However, to catch up with increasing network traffic, it should shift from a type of the homogeneous network with a single tier to a type of the heterogeneous network (HetNet) with multiple tiers, where the HetNet is composed of different types of networks. It is a popular and efficient way to improve the network capacity such as the coexistence system between WiFi and LTE. This changing trend in wireless networks demands a new look on how to design the associations of uplink and downlink in the coexistence system.

Obviously, the coupled associations of uplink and downlink in HetNets, will lead to asymmetric traffic between the uplink and downlink in WiFi and LTE coexistence system. But with the rapid growth of real-time applications, video-calling, and online social networking, the fairness between uplink and downlink is becoming more and more important and necessary. Therefore, the concept of DUDe has been proposed to deal with this problem, which represented an innovative association way to improve the whole network performance [26]. As a consequence, in this paper, we consider to use DUDe in WiFi and LTE coexistence system. The transmissions for uplink and downlink will be independent while DUDe has already been supported by the existing LTE specifications. Therefore, from a theoretical point of view, the DUDe can be also accomplished in the coexistence system. In this paper, we assume the LTE BS and WiFi APs have the same service provider and a cloud server is deployed at the back-end. The links between them are wired so that the delay can be ignored. The communication 


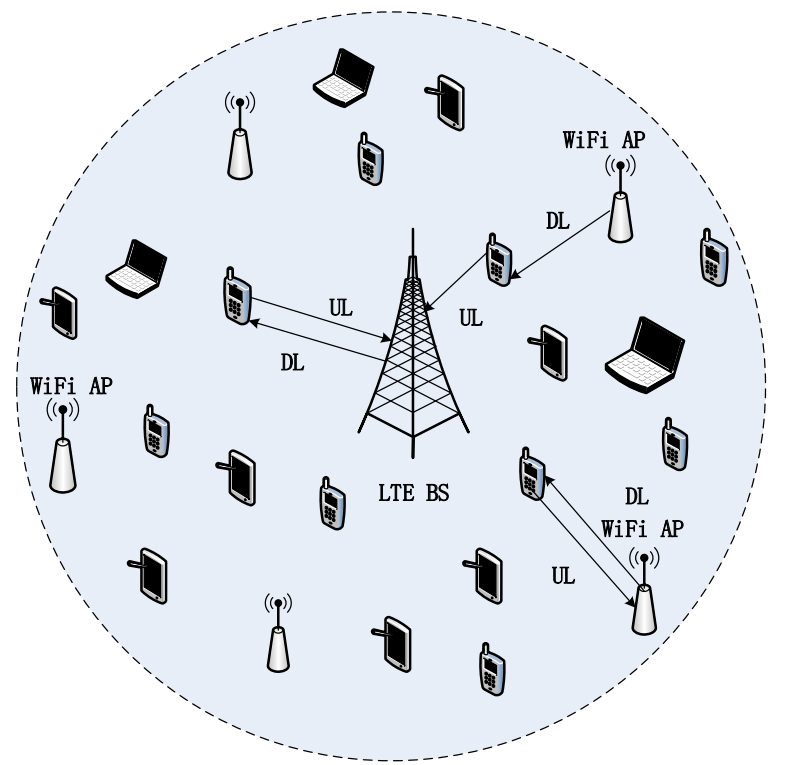

Fig. 1: Decoupling for WiFi and LTE coexistence system in a specific area.

process can be expressed as follows. If a user sends an acknowledgement (ACK) for service request to LTE BS, BS will transfer the ACK to the cloud server, which is capable of powerful computation. The cloud server can calculate the optimal solution to guarantee both the total throughputs and load balance between uplink and downlink based on all the inputs from WiFi and LTE. Then, it will send the schemes of users' association and the plan of bandwidth allocation back to the BS or APs. Finally, the BS or APs will send an ACK to the users that they will provide service for. To sum up, applying DUDe in WiFi and LTE coexistence system is feasible.

The benefits of DUDe in WiFi and LTE coexistence system are obvious and evident. DUDe in WiFi and LTE coexistence system can be supported by the existing network architecture so that there are no big changes in the design and implementation of networks and hardware facilities. And the users of WiFi and LTE coexistence system can choose which network to access based on their own requirements. For example, if a user needs stable transmission in the uplink and highspeed transmission in the downlink, it can choose to access BS in uplink and AP in downlink. Furthermore, the fairness between uplink and downlink can be greatly improved by DUDe. At last, it can decrease the interference in WiFi and LTE coexistence system.

\section{MODELING AND FORMULATION}

In this section, we first introduce the system model. Then, we analyze the throughputs of uplink and downlink. Finally, we will give the formulation of our problem of proportional fairness.

\section{A. Coexistence system model}

The coexistence system model is considered in Fig. 1, consisting of one LTE BS and several WiFi APs. One BS is located at the center of the geographical area while multiple
WiFi APs are covered by the BS. Under this coexistence architecture, we denote $\mathcal{K}$ as the group of WiFi APs and $L$ as the BS of LTE. Denote $\mathcal{N}$ as the group of users within BS and APs, who hope to access this network. The LTE system is designed to support orthogonal frequency division multiplexing access (OFDMA) transmission. The WiFi network operates with the current CSMA/CA protocol and all users contend to access the whole channels for transmission opportunities. Here, a specific unlicensed band is shared by the LTE network and the WiFi network, where the bandwidth is $B$ and is divided into $B_{L}$ and $B_{W}$ for LTE and $\mathrm{WiFi}$, respectively. Since there is no overlap between $B_{W}$ and $B_{L}$, the interference between $\mathrm{WiFi}$ and LTE can be completely avoided. Therefore, the bandwidth allocation can be expressed as:

$$
B_{W}+B_{L}=B
$$

where $B_{W}$ and $B_{L}$ are continuous variables and can be adjusted with the current situation of the network. In order to maximize the throughputs of uplink and downlink simultaneously with load balance, we assume that the uplink and downlink could be decoupled. It means that each user is allowed to choose any network to access its own uplink or downlink.

To model one user's access status for its uplink and downlink, we denote binary decision variables $x_{i L}^{U}$ and $x_{i L}^{D}$ as whether user $i(i \in \mathcal{N})$ chooses LTE BS, i.e.,

$$
\begin{aligned}
& x_{i L}^{U}= \begin{cases}1 & \text { If user } i \text { chooses LTE BS to provide } \\
\text { its service in the uplink; } & \text { else. }\end{cases} \\
& x_{i L}^{D}= \begin{cases}1 & \text { If user } i \text { chooses LTE BS to provide } \\
\text { its service in the downlink; } & \text { else. }\end{cases}
\end{aligned}
$$

Similarly, we denote binary decision variables $y_{i j}^{U}$ and $y_{i j}^{D}$ as whether user $i(i \in \mathcal{N})$ accesses $\mathrm{WiFi}$ AP $j(j \in \mathcal{K})$, i.e.,

$y_{i j}^{U}= \begin{cases}1 & \text { If user } i \text { chooses WiFi AP } j \text { to provide } \\ & \text { its service in the uplink; } \\ 0 & \text { else. }\end{cases}$

$y_{i j}^{D}= \begin{cases}1 & \text { If user } i \text { chooses WiFi AP } j \text { to provide } \\ & \text { its service in the downlink; } \\ 0 & \text { else. }\end{cases}$

Therefore, all the users in $\mathrm{WiFi}$ and LTE coexistence network are divided into four groups: (I) Users attached to both the uplink and downlink of BS, (II) Users attached to the uplink of AP and the downlink of BS, (III) Users attached to the uplink of BS and the downlink of AP, and (IV) Users attached to both the uplink and downlink of AP. Since the uplink or downlink of a user can be served by either LTE BS or one of WiFi APs, for each user $i$, we have:

$$
\begin{aligned}
& x_{i L}^{U}+\sum_{j \in \mathcal{K}} y_{i j}^{U} \leq 1, \\
& x_{i L}^{D}+\sum_{j \in \mathcal{K}} y_{i j}^{D} \leq 1 .
\end{aligned}
$$

We assume that the channel in our model is Rayleigh channel. When the signal $s(t)$ of power $P$ is transmitted on 
the Rayleigh channel, the received signal $r(t)$ can be obtained as $r(t)=h(t) l(t) s(t)+n(t)$, where $n(t) \sim N\left(0, \sigma_{n}^{2}\right)$ is the white Gaussian noise (WGN). $l(t)=d^{-\alpha}$ is the amplitude attenuation caused by path loss, where $d$ is the distance from transmitter to receiver, $2 \leq \alpha \leq 4$ is the factor of path loss. $l(t)$ is a constant while the distance is fixed. $h(t)$ is the channel gain and obeys independent and identically distributed (i.i.d) Rayleigh distribution, which means $h(t)$ changes randomly at different time slots. And its probability density function is $f_{h}(s)=\frac{s}{\sigma^{2}} e^{-\frac{s^{2}}{2 \sigma^{2}}}, s \geq 0$. Hence, at time $t$, the actual received signal power can be obtained as $P_{r}(t)=\gamma(t) P d^{-\alpha}=$ $h^{2}(t) P d^{-\alpha}$, where $h^{2}(t)$ obeys exponential distribution and its probability density function is $f_{\gamma}(s)=\frac{1}{2 \sigma^{2}} e^{-\frac{s^{2}}{2 \sigma^{2}}}, s \geq 0$. Based on the above analysis, at time $t$, the SNR of the channel can be expressed as $S N R=\frac{h^{2}(t) P d^{-\alpha}}{\sigma_{n}^{2}}$.

\section{B. WiFi Throughput Analysis}

Assuming that each user is under the coverage of at least one AP $j$, we model the coverage of one WiFi AP by defining $\mathcal{A}_{i}$ as: (1) the set of users covered by a WiFi AP $i$, or (2) the set of WiFi APs covering the user $i$. For simplicity, we assume each user's traffic is saturated. Hence, we can apply the empirical throughput model in [29] to our formulation. With CSMA/CA, all the users and APs have the same probabilities to access the channel. For user $i$, who is served by the WiFi $\mathrm{AP} j$, it should contend the channel access with other WiFi users in $\mathcal{N}_{i}$ and the WiFi APs in $\mathcal{W}_{i}$ within its contention range.

Note that, if there is any user in downlink access WiFi AP $j$, it indicates that $\mathrm{AP} j$ is active, which can be expressed as $\sum_{i \in \mathcal{A}_{j}} y_{i j}^{D} \neq 0$. We define a new variable $K_{j}$ as whether the $\mathrm{WiFi} \mathrm{AP} \mathrm{j}$ is active or not. That is,

$$
K_{j}= \begin{cases}1 & \text { If } \sum_{i \in \mathcal{A}_{j}} y_{i j}^{D} \neq 0 \\ 0 & \text { else }\end{cases}
$$

where $j \in \mathcal{W}_{i}$. Based on the above discussion, we can express the total number of active users' uplink and WiFi APs that is within user $i$ 's CSMA contention range as follows:

$$
M_{i}=\sum_{k \in \mathcal{N}_{i}} \sum_{j \in \mathcal{A}_{k}} y_{k j}^{U}+\sum_{j \in \mathcal{W}_{i}} K_{j} .
$$

Therefore, if user $i$ 's uplink is served by the WiFi networks, the channel access time is shared equally by $M_{i}$. And the achievable throughput for user $i$ 's uplink can be obtained as follows:

$$
r_{W, i j}^{U}=\frac{\varepsilon_{i}}{M_{i}+1} y_{i j}^{U} B_{W} \log _{2}\left(1+\frac{Q_{W, i} d_{i j}^{-\alpha} h_{i j}^{2}}{\sigma_{n}^{2}}\right),
$$

where $\varepsilon_{i}$ is the channel efficiency, $Q_{W, i}$ is the power spectral density (PSD) of user $i, d_{i j}$ is the distance between user $i$ and AP $j, \alpha$ is the factor of path loss, $h_{i j}$ is the channel gain, and $\sigma_{n}^{2}$ is the Gaussian PSD.

Now we discuss the achievable downlink throughput of user $i$. User $i$ is served by $\mathrm{WiFi} \mathrm{AP} j$. WiFi AP $j$ performs the same rule as a user to access the channel by contending with users and other WiFi APs, who are all within $j$ 's CSMA contention range. Therefore, the channel access time for a WiFi AP $j$ is $\frac{1}{M_{j}+1}$. We assume the WiFi AP $j$ equally shares the channel access time to its users' downlinks for service. Then the throughput of user $i$ for the downlink can be obtained:

$$
\begin{aligned}
r_{W, i j}^{D}= & y_{i j}^{D} \frac{1}{M_{j}+1} \frac{1}{\sum_{k \in \mathcal{A}_{j}}^{k \neq i} y_{k j}^{D}+1} \\
& \cdot \varepsilon_{i} B_{W} \log _{2}\left(1+\frac{Q_{W, i} d_{i j}^{-\alpha} h_{i j}^{2}}{\sigma_{n}^{2}}\right) .
\end{aligned}
$$

The throughput in (10) and (11) can only represent one user's uplink and downlink throughput, respectively. And the total uplink and downlink throughput of WiFi system are as follows, respectively:

$$
\begin{aligned}
& r_{W}^{U}=\sum_{i \in \mathcal{N}} \sum_{j \in \mathcal{K}} r_{W, i j}^{U}, \\
& r_{W}^{D}=\sum_{i \in \mathcal{N}} \sum_{j \in \mathcal{K}} r_{W, i j}^{D} .
\end{aligned}
$$

To guarantee there is a minimum bandwidth for the WiFi users, the minimum bandwidth requirement of WiFi system can be assumed as $B_{\min }^{W}$. Then the bandwidth constraint is:

$$
B_{W} \geq B_{\min }^{W}
$$

\section{LTE Throughput Analysis}

LTE BS has a central management to divide its bandwidth $B_{L}$ into a group of distinct channels for its users. Denote $B_{i}^{U}$ as the bandwidth allocated to the uplink of user $i$ as well as $B_{i}^{D}$ to the downlink of user $i$. To ensure the performance of LTE network, we denote $B_{\min }^{L}$ as the minimum bandwidth allocated to one user's uplink or downlink, which is served by LTE BS. Then, we have:

$$
\begin{aligned}
& x_{i L}^{U} \cdot B_{\min }^{L} \leq B_{i}^{U} \leq x_{i L}^{U} \cdot B_{L}, \\
& x_{i L}^{D} \cdot B_{\min }^{L} \leq B_{i}^{D} \leq x_{i L}^{D} \cdot B_{L},
\end{aligned}
$$

And in order to maximize spectrum efficiency, no more bandwidth will be allocated to LTE system in excess of their demands. Then, we have:

$$
\sum_{i \in \mathcal{N}}\left(x_{i L}^{U} \cdot B_{i}^{U}+x_{i L}^{D} \cdot B_{i}^{D}\right)=B_{L} .
$$

Therefore, the achievable throughput for user $i$ 's uplink can be expressed as follows:

$$
r_{L, i}^{U}=x_{i L}^{U} \cdot B_{i}^{U} \log _{2}\left(1+\frac{Q_{L, i} d_{i L}^{-\alpha} h_{i L}^{2}}{\sigma_{n}^{2}}\right),
$$

where $Q_{L, i}$ is the PSD for user $i$ under LTE, $d_{i L}$ is the distance between user $i$ and LTE BS, $\alpha$ is the factor of path loss, $h_{i L}$ is the channel gain and $\sigma_{n}^{2}$ is the Gaussian PSD. And the achievable throughput of user $i$ 's downlink is:

$$
r_{L, i}^{D}=x_{i L}^{D} \cdot B_{i}^{D} \log _{2}\left(1+\frac{Q_{L, i} d_{i L}^{-\alpha} h_{i L}^{2}}{\sigma_{n}^{2}}\right) .
$$

Therefore, the total uplink and downlink throughput of LTE system are as follows, respectively:

$$
\begin{aligned}
& r_{L}^{U}=\sum_{i \in \mathcal{N}} r_{L, i}^{U}, \\
& r_{L}^{D}=\sum_{i \in \mathcal{N}} r_{L, i}^{D} .
\end{aligned}
$$




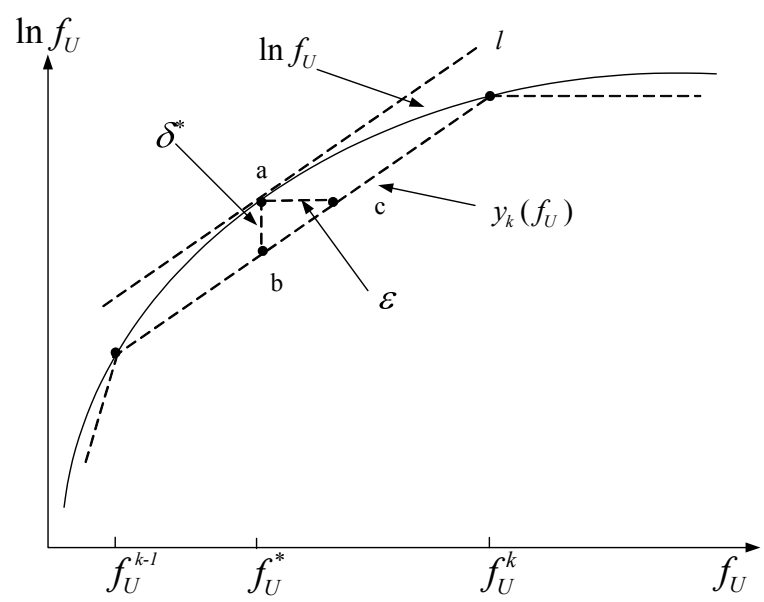

Fig. 2: An illustration of piece-wise linear approximation for $\log$ function.

\section{Problem Formulation}

With the aim of achieving the relatively fair throughput between uplink and downlink in the coexistence system, we introduce a utility function $U(S)$, which is widely used to achieve proportional fairness in various systems [23], [30], and [31], i.e.,

$$
U(S)=\ln (S),
$$

where $\ln (\cdot)$ denotes the natural logarithmic (log) function. In order to realize both the sum throughput and load balance, a utility function $F$ for the coexistence network can be defined as:

$$
F=\ln \left(r_{W}^{U}+r_{L}^{U}\right)+\ln \left(r_{W}^{D}+r_{L}^{D}\right),
$$

where $\left(r_{W}^{U}+r_{L}^{U}\right)$ is the whole uplink throughput of the coexistence network and $\left(r_{W}^{D}+r_{L}^{D}\right)$ is the whole downlink throughput of the coexistence network. Thus, an optimization framework can be formulated as follows:

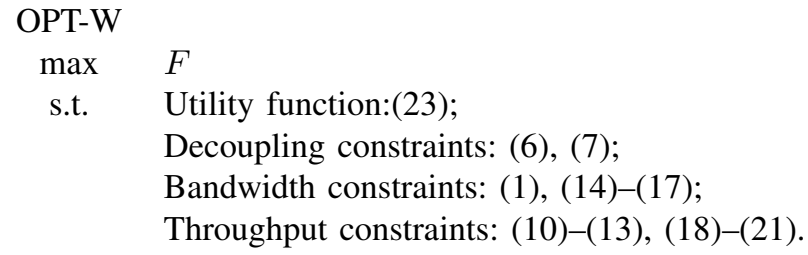

In this formulation, $x_{i L}^{U}, x_{i L}^{D}, y_{i j}^{U}$ and $y_{i j}^{D}$ are binary variables, $K_{j}$ and $M_{i}$ are integer variables, $B_{W}, B_{L}, B_{i}^{U}, B_{i}^{D}, r_{W, i j}^{U}$, $r_{W, i j}^{D}, r_{L, i j}^{U}, r_{L, i j}^{D}, r_{L}^{U}, r_{L}^{D}, r_{W}^{U}, r_{W}^{D}$ and $F$ are continuous variables. Since there are not only the product terms but also the $\log$ function terms in constraint of this formulation, this optimization problem is obvious of a mixed-integer Non-linear program (MINLP). In general, it is NP-hard [32].

\section{E. Reformulation}

In this subsection, we transform the nonlinear constraints in OPT-W into the linear ones. Our first step is to employ Reformulation-Linearization technique (RLT) [32] to remove the nonlinear terms in OPT-W without loss of optimality. Then we substitute the log function term in (23) with a set of linear constraints using piece-wise linear approximation technique.
After these two steps, we will have a linearized problem OPT$\mathrm{S}$, which can be solved by the software named CPLEX.

In the above formulation, constraints (10), (11), (15) - (19) are nonlinear. We show how to transform them into a series of linear constraints.

In (15)-(19), we have nonlinear terms $x_{i L}^{U} B_{i}^{U}, x_{i L}^{D} B_{i}^{D}$, $x_{i L}^{U} B_{L}$ and $x_{i L}^{D} B_{L}$. Taking the term $x_{i L}^{U} B_{i}^{U}$ as an example and defining $z_{i}^{U}=x_{i L}^{U} B_{i}^{U}$, we have the following associate constraints:

$$
\begin{aligned}
& x_{i L}^{U} \geq 0, \quad 1-x_{i L}^{U} \geq 0 . \\
& B_{i}^{U} \geq 0, \quad B-B_{i}^{U} \geq 0 .
\end{aligned}
$$

The two constraints involving $x_{i L}^{U}$ can be cross-multiplied with the two constraints involving $B_{i}^{U}$. The product term $x_{i L}^{U} B_{i}^{U}$ can be substituted with $z_{i}^{U}$. Then, $x_{i L}^{U} B_{i}^{U}$ can be substituted by the following linear constraints:

$$
\begin{gathered}
z_{i}^{U} \geq 0 \\
z_{i}^{U} \leq x_{i L}^{U} B, \\
z_{i}^{U} \leq B_{i}^{U}, \\
z_{i}^{U} \geq x_{i L}^{U} B+B_{i}^{U}-B,
\end{gathered}
$$

where $i \in \mathcal{N}$.

The same can be done for $x_{i L}^{D} B_{i}^{D}, x_{i L}^{U} B_{L}$ and $x_{i L}^{D} B_{L}$. Define $z_{i}^{D}=x_{i L}^{D} B_{i}^{D}, A_{i}^{U}=x_{i L}^{U} B_{L}$ and $A_{i}^{D}=x_{i L}^{D} B_{L}$. Therefore, (15), (16), (17), (18) and (19) can be replaced by a series of linear constraints for $z_{i}^{U}, z_{i}^{D}, A_{i}^{U}$ and $A_{i}^{D}$, respectively. To simplify, we omit these similar processing with RLT and give them in Appendix A.

We can rewrite the constraint (10) into the following form:

$$
M_{i} \cdot r_{W, i j}^{U}+r_{W, i j}^{U}=\varepsilon_{i} \cdot y_{i j}^{U} B_{W} \log _{2}\left(1+\frac{Q_{W, i} d_{i j}^{-\alpha} h_{i j}^{2}}{\sigma_{n}^{2}}\right) .
$$

By defining $D_{i j}=M_{i} r_{W, i j}^{U}$ and $\mu_{i j}^{U}=y_{i j}^{U} B_{W}$, we can similarly replace (10) by a series of linear constraints for $D_{i j}$ and $\mu_{i j}^{U}$ in Appendix B.

Also, the similar processing can be done for (11). Define the new variables $\theta_{i j}=\sum_{k \in \mathcal{A}_{j}}^{k \neq i} y_{k j}^{D} r_{W, i j}^{D}$ and $\mu_{i j}^{D}=y_{i j}^{D} B_{W}$. Then, we have

$$
\left(\theta_{i j}+r_{W, i j}^{D}\right)\left(M_{j}+1\right)=\mu_{i j}^{D} \varepsilon_{i} \log _{2}\left(1+\frac{Q_{W, i} d_{i j}^{-\alpha} h_{i j}^{2}}{\sigma_{n}^{2}}\right) .
$$

By defining $\tau_{i j}=\theta_{i j}+r_{W, i j}^{D}$ and $\zeta_{i j}=\tau_{i j} M_{j}$, we can similarly replace (11) by a series of linear constraints for $\theta_{i j}$, $\mu_{i j}^{D}, \tau_{i j}$ and $\zeta_{i j}$ in Appendix C.

For (23), we employ a piece-wise linear approximation technique in [33] to transform the nonlinear term inside the logarithmic function into the linear one. Through introducing the new variables $f_{U}$ and $f_{D}$, the following constraints can be obtained:

$$
F=\ln \left(f_{U}\right)+\ln \left(f_{D}\right) \text {. }
$$

Taking $\ln \left(f_{U}\right)$ as an example, the idea is to use a set of finite $M$ line segments for the approximation of the log function with guaranteed performance as shown in Fig. 2.

We denote $f_{U}^{\min }$ and $f_{U}^{\max }$ as the lower and upper bounds of $f_{U}$, respectively. We define the value of 


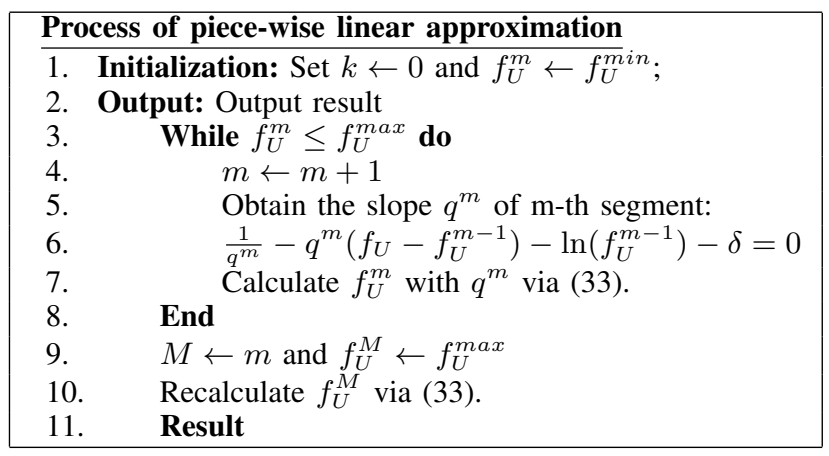

Fig. 3: An algorithm to linearize the nonlinear term inside log function.

$f_{U}^{0}, f_{U}^{1}, \cdots, f_{U}^{m}, \cdots, f_{U}^{M}$ on the horizontal axis as the end point of $M$ segments, where $f_{U}^{\min }=f_{U}^{0}, f_{U}^{\max }=f_{U}^{M}$.

The minimum number of line segments $M$ can be found through the iterative process as follows, where our algorithm of the linearizing technology is shown in Fig. 3. Starting from $f_{U}^{0}$, we should calculate the slope of the first segment while guaranteeing that the requirement of approximation error $\delta$ can be achieved. Then, based on this determined slope, we can find the second point $f_{U}^{1}$ on the horizontal axis, which represents the right-side end point of the first segment. From the second point $f_{U}^{1}$, we can repeat the similar process for the next one and so forth. Once the value of last point exceeds $f_{U}^{\max }$, the whole process will be terminated.

Specially, we denote slope of the m-th linear segment as $q^{m}$, i.e.,

$$
q^{m}=\frac{\ln \left(f_{U}^{m+1}\right)-\ln \left(f_{U}^{m}\right)}{f_{U}^{m+1}-f_{U}^{m}} .
$$

Denote $y_{m}\left(f_{U}\right)$ as the $\mathrm{m}$-th linear segment that approximates $\ln \left(f_{U}\right)$ and it can be expressed as:

$$
y_{m}\left(f_{U}\right)=q^{m}\left(f_{U}-f_{U}^{m-1}\right)+\ln \left(f_{U}^{m}\right) .
$$

For a given error bound $\delta$, we can iteratively find the values of $f_{U}^{0}, f_{U}^{1}, \cdots, f_{U}^{m}, \cdots, f_{U}^{M}$ and slopes $q^{1}, q^{2}, \cdots, q^{m}, \cdots, q^{M}$ by the above piece-wise linear process as shown in Fig. 3. Meanwhile, we can obtain the values of $f_{U}^{m}$ and $q^{m}$ by exploiting numerical methods like Newton's method or bisection method as in [34]. The same is for $\ln \left(f_{D}\right)$, assuming the slope is $p^{m}$. Then, we can substitute utility function $F$ with the following linear constraints:

$$
\begin{gathered}
F=y_{m}\left(f_{U}\right)+y_{m}\left(f_{D}\right), \\
y_{m}\left(f_{U}\right) \leq q^{m}\left(f_{U}-f_{U}^{m-1}\right)+\ln \left(f_{U}^{m}\right), \\
y_{m}\left(f_{D}\right) \leq p^{m}\left(f_{D}-f_{D}^{m-1}\right)+\ln \left(f_{D}^{m}\right),
\end{gathered}
$$

where $m=1,2, \cdots, M$. Therefore, the OPT-W can be reformulated into a new optimization problem OPT-S.

\section{OPT-S}

$\max F$

s.t. Utility function: (35)-(37);

Decoupling constraints: (6), (7);

Bandwidth constraints: (1), (14), (15)-(17);

Throughput constraints: (10)-(13), (18)-(21).
Note that, in the formulation of OPT-W problem, (10), (11), (15)-(19) are already the linear constraints, which can be replaced with a series of linear constraints in Appendix A, B and $\mathrm{C}$. Obviously, this optimization problem is a mixed-integer linear program (MILP), in general, which is still NP-hard [32]. And it can be easily solved by CPLEX.

\section{USERS' Number PREDICTION}

In order to make our research more realistic, we plan to use machine learning algorithm based on the regression model for predicting the total number of users in the coexistent networks between WiFi and LTE, where the predicted result can be used in the formulation of the above fairness problem with DUDe. It seems that deep learning algorithm and other machine learning algorithms show great potential in optimization of wireless communications [35]-[40]. However, as a traditional method with low complexity, SVM is still an effective and attractive algorithm with high prediction accuracy to extensively predict future system characteristics, which can be referred to in [28]. Therefore, in this paper, we will apply SVM to predict the total number of users, which can enable not only static analysis but also dynamic analysis of the coexistence system. As we all know, dynamic traffic prediction or analysis is useful for predictive network traffic control, resource management and network management in wireless networks. To simplify, we will focus on the total number of users in coexistence network at a specific time in the future, which can be predicted by the proposed SVM algorithm. Under this case, based on the predictions of total number of users and the associations of users' behavior, we can not only allocate appropriate spectrum resources for the coexistence system but also obtain load balance between uplink and downlink in the future.

SVM has strong non-linear processing ability in regression, which is mainly used in system modeling, time series prediction and optimal control [41], [42]. As for time series prediction, SVM can be used for stock prediction, Internet traffic prediction, electricity prediction and many other applications [43], [44], [46]-[53].

\section{A. Prediction Problem Definition}

In order to predict the total number of users in WiFi and LTE coexistence network, we describe the prediction as a stochastic process: $N=\left(n_{t}: t=0,1,2, \cdots\right)$. And the prediction problem can be expressed as: given the current and the past total number of users $N_{t}=\left(n_{t-p+1}, \ldots, n_{t-1}, n_{t}\right)$ to predict the total number of users in the future $n_{t+q}$, where $p$ is the length of past data used for forecasting and $q$ is step value for forecasting.

\section{B. SVM Regression Model}

Via a nonlinear mapping, SVM can reflect the data into a higher-dimensional feature space. Then, it fulfills linear regression in the feature space. Assuming $w_{i}$ as the input vector and $v_{i}$ as the desired value, we can define the training data set as $N=\left(w_{i}, v_{i}\right) \in R^{n} \times R$ with $i=1,2, \cdots$. Then, for the regression problem, if we can determine a function 
$f(w)$, it can accurately approximate the future values. Assuming $\omega$ as the weight vector, $\phi$ as a non-linear transformation form, and $b$ as the threshold, we can define the function $f(w)=\omega \cdot \phi(w)+b$. And further introducing the insensitive loss function $\varepsilon$, the SVM regression can be expressed as follows:

$$
\begin{aligned}
\min \quad \frac{1}{2}\left\|\omega^{2}\right\|+C \sum_{i=1}^{l}\left(\xi_{i}+\xi_{i}^{*}\right) \\
\text { s.t. } \quad v_{i}-\omega \cdot \phi(w)-b \leq \varepsilon+\xi_{i}, \xi_{i} \geq 0 \\
\omega \cdot \phi(w)+b-v_{i} \leq \varepsilon+\xi_{i}^{*}, \xi_{i}^{*} \geq 0
\end{aligned}
$$

where $C \geq 0$ is a constant to represent the tradeoff between the complexity of regression model and training error, $\xi_{i}$ and $\xi_{i}^{*}$ are the slack variables. By using duality principle and introducing Lagrange multipliers, (38) can be transferred into its dual problem:

$$
\begin{array}{ll}
\max & -\frac{1}{2} \sum_{i=1}^{l} \sum_{j=1}^{l}\left(\alpha_{i}-\alpha_{i}^{*}\right)\left(\alpha_{j}-\alpha_{j}^{*}\right) K\left(w_{i}, w_{j}\right) \\
& -\varepsilon \sum_{i=1}^{l}\left(\alpha_{i}+\alpha_{i}^{*}\right)+\sum_{i=1}^{l} v_{i}\left(\alpha_{i}-\alpha_{i}^{*}\right) \\
& \text { s.t. } \quad \sum_{i=1}^{l}\left(\alpha_{i}-\alpha_{i}^{*}\right)=0 \\
& C \geq \alpha_{i}, \alpha_{i}^{*} \geq 0
\end{array}
$$

where $K\left(w_{i}, w_{j}\right)=\phi\left(w_{i}\right) \cdot \phi\left(w_{j}\right)$ is defined as the kernel function. Its advantages lie in calculation of kernel function in low-dimensional space rather than operation on inner product in high-dimensional space. Therefore, it avoids the disaster of dimensionality and the explicit calculation of $\phi(w)$. The most commonly used kernel function of SVM is radial basis function (RBF): $K\left(w_{i}, w_{j}\right)=\exp \left(-\left\|w_{i}-w_{j}\right\|^{2} / 2 \sigma^{2}\right)$, where $\sigma$ is the variance to show a degree for spread around a mean in statistics. According to the relationship between the parameters $\gamma$ and $\sigma$ with $\gamma=1 / 2 \sigma^{2}$, we can obtain the equivalent RBF kernel function of SVM $K\left(w_{i}, w_{j}\right)=\exp \left(-\gamma\left\|w_{i}-w_{j}\right\|^{2}\right)$ [54], [55]. The parameter $\gamma$ is the inverse of the influence radius of samples selected as support vectors in SVM. It is important to show how far the influence of training sample can achieve.

In this paper, a set of training samples $D=(W, V)$ are first constructed. The input of $D$ is $W=\left(n_{t-p+1}, \cdots, n_{t-1}, n_{t}\right)$ and the output of $D$ is $V=n_{t+q}$, where $t=1,2, \cdots, T$ and $T$ is the amount of sample $D$. Then, on the given training set, an SVM is training to predict the future number value of users in the coexistence network.

Besides, in order to evaluate the performance of prediction, mean square error (MSE) and normalized mean square error (NMSE) can be taken used in this SVM scheme. With the increase of prediction accuracy, the MSE will be smaller, which can be widely used to evaluate the performance of prediction. Further, the value of NMSE will be 0 for a perfect predictor; it will be 1 for a trivial predictor, where the mean of
TABLE II: Parameters section for prediction of number of users.

\begin{tabular}{ccc}
\hline$\varepsilon$ & $\sigma$ & $C$ \\
\hline 0.0001 & 0.4 & 1 \\
\hline
\end{tabular}

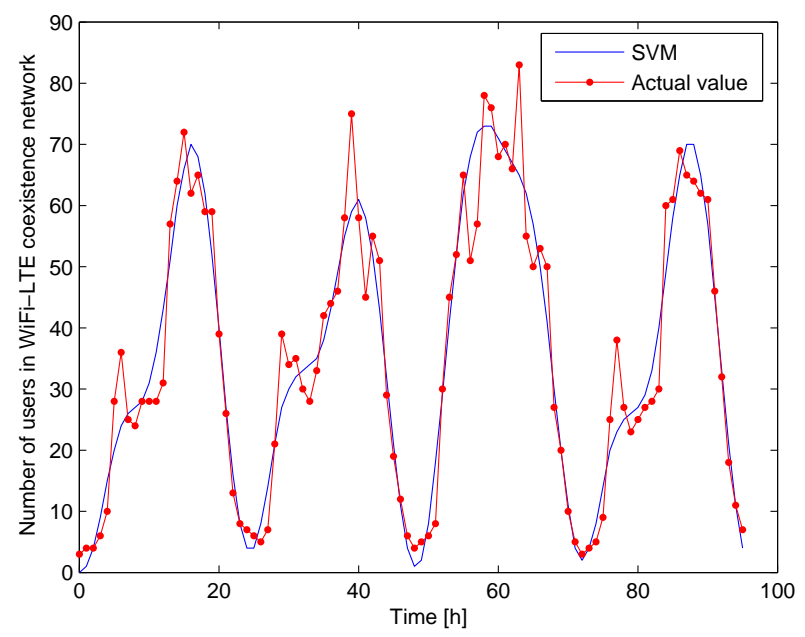

Fig. 4: Prediction result.

the actual time series can be statistically forecasted; it may be greater than 1, where the performance of prediction is worse than that of the trivial predictor.

\section{Experiments on Prediction with SVM}

The experiments on prediction with SVM are accomplished by using a real-life dataset, which is obtained from a small community network. What kernel function will be selected in SVM is vital while applying SVM to predict the total number of users in the coexistence network. In our experiment, Gaussian RBF is the first selection for the kernel function of $\mathrm{SVM}$. Then, the parameters $(\varepsilon, \sigma$ and $C$ ) in SVM regression model should be determined, which affect the estimation accuracy of SVM. As for the parameter $\varepsilon$, it represents the range of error insensitivity and reflects the noise range in the observed time series of user's number. As for the parameter $\sigma$, it is the variance to show the capacity of the learning machine. When $\sigma$ is quite small, the capacity is strong. As for the parameter $C$, it is the penalty coefficient to tradeoff the complexity with the training error of the regression model. In order to train the regression for minimum error with lower generalization, higher penalties should be assigned with larger $C$ to errors. Otherwise, fewer penalties will be assigned with smaller $C$ to errors. In this paper, based on priori knowledge [45], we can select these parameters which are shown in Table II.

For SVM-based prediction method, in fact, we apply 144 training samples to predict the next 96 samples. And the performance of prediction can be seen in details in Fig. 4, where Time means the variable or $\mathrm{x}$ label, and $\mathrm{h}$ means the unit of Time (i. e., hour). Simulation results can prove that SVM based prediction possesses the advantages of high prediction 


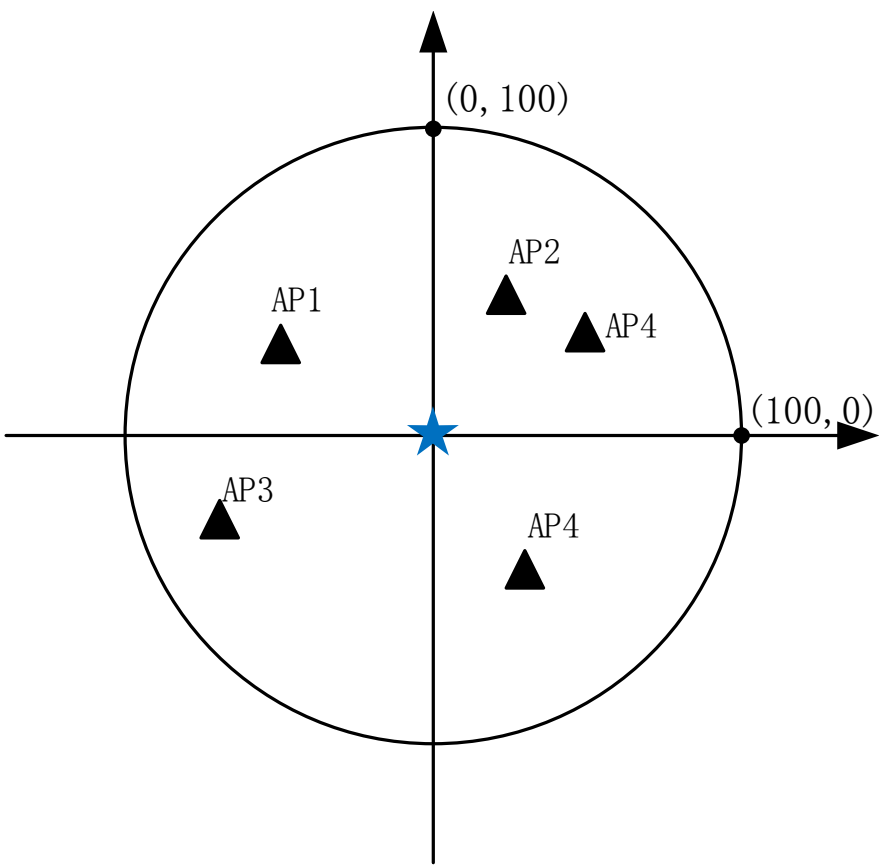

Fig. 5: Random deployment of LTE BS and WiFi APs in a circle.

accuracy with small approximation error on each single point of it, as well as its low complexity in calculations.

\section{Simulation Results}

In this section, we will first discuss the simulation results to investigate the performance of the decoupling mechanism for WiFi and LTE coexistence system. Firstly, the simulation parameters are introduced. Then, the performances of decoupling based on prediction are evaluated. Finally, the improvements in terms of the sum rate and the fairness between uplink and downlink will be displayed, by comparing the proposed decoupling scheme with coupled coexistence scheme with associations.

\section{A. Parameter Setting}

Within a circular area with radius 100 meters, one LTE BS and multiple WiFi APs are considered to randomly deploy. The locations of one BS and five WiFi APs are shown in Fig. 5. In general, we normalize the units for bandwidth, distance, power and throughput with appropriate dimension. The transmission ranges of LTE BS and WiFi APs are set to 100 meters and 40 meters, respectively. We also set the CSMA contention range for WiFi to be 60 meters. The total bandwidth in the unlicensed spectrum to be used is with $B=100 \mathrm{MHz}$. The minimum bandwidth remained for WiFi network is with $B_{m i n}^{W}=10 \mathrm{MHz}$ while coexisting with LTE. The transmission PSDs for each user under WiFi and LTE are set to 1.0 and 3.0 , respectively. The ambient Gaussian PSD is $\sigma_{n}^{2}=10^{-6}$ $\mathrm{W} / \mathrm{Hz}$. The factor of path $\operatorname{loss} \alpha$ is 2 . The antenna gains are set to 1 between the user and WiFi AP and 2 between the user and LTE BS. And the channel efficiency for WiFi is assumed as $70 \%$.

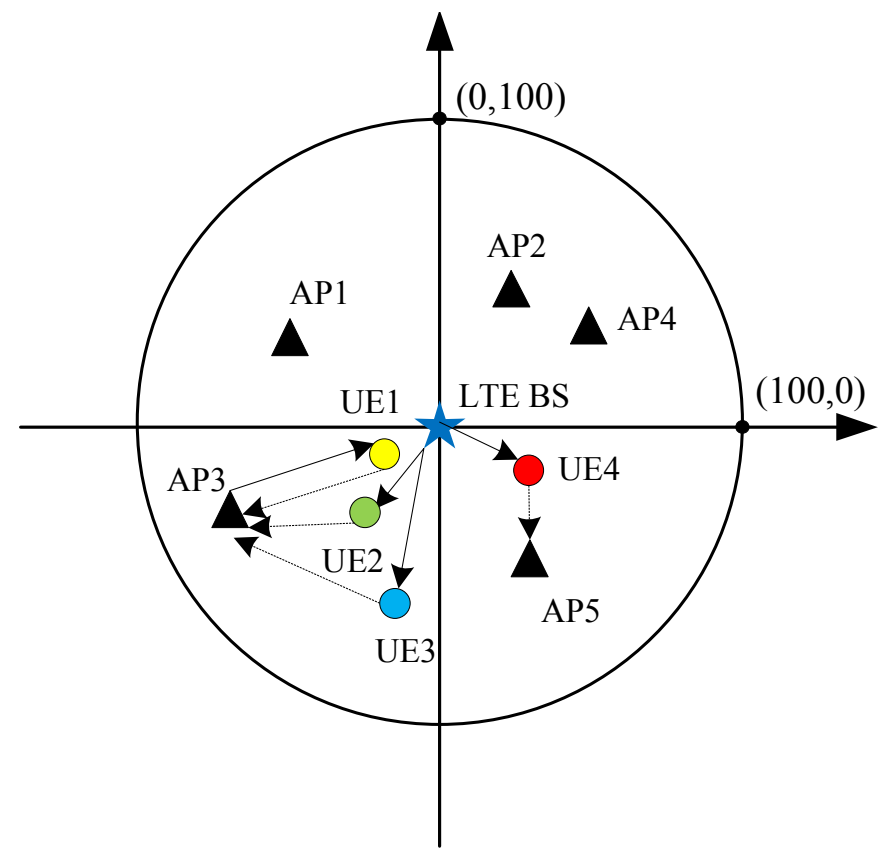

Fig. 6: Four users' association status in WiFi and LTE coexistence network with DUDe.

\section{B. Rate Comparisons between coupling and decoupling}

We assume the total number of users continuously changes by our prediction. The users will be randomly deployed within the circular area. Our simulation time is 96 hours. Under the circumstances of WiFi and LTE coexistence, we push forward the simulations with and without DUDe, respectively. We also compare the throughput ratios between uplink and downlink, respectively, and the total system throughput under PF objective.

In order to show users' association status in WiFi and LTE coexistence network with DUDe, we take the results of the fourth predicted moment as an example. The predicted total number of users in the network and users' associations at this time are shown in Fig. 6.

Fig. 7 and Fig. 8 show the comparison of ratios of uplinkdownlink throughput in $\mathrm{WiFi}$ and LTE coexistence network with and without DUDe, which are changed by time and users' number, respectively. We can find that at any time, the ratio of decoupling status is closer to 1 than that in coupled case, which means that DUDe achieves load balance between uplink and downlink. In Fig. 7, there are two moments that the ratio is 0 when the WiFi and LTE coexistence network is without DUDe. They indicate that in the coupled case, the throughput of the uplink and downlink has been seriously unbalanced, and even the uplink throughput is 0 .

Besides, as shown in Fig. 8, while the total number of users increases, the unfair situation of uplink and downlink will become serious both in the decoupling and coupled networks. The reason for this situation is due to the distributed coordination function (DCF) mechanism of WiFi network, where the probability of WiFi APs and users accessing channels is the same. Therefore, while the total number of users increases, the probability of uplink and downlink accessing the 


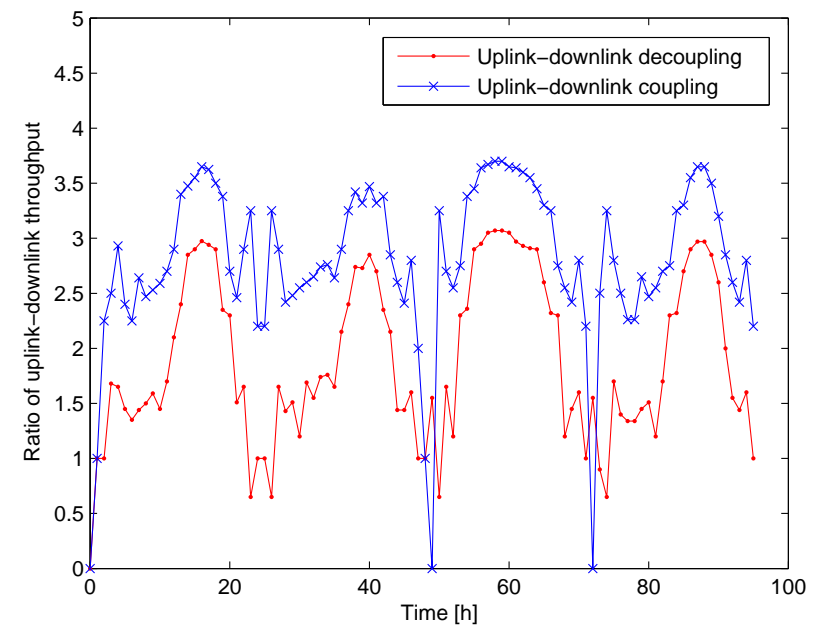

Fig. 7: Ratios of uplink-downlink throughput in WiFi and LTE coexistence network with and without DUDe.

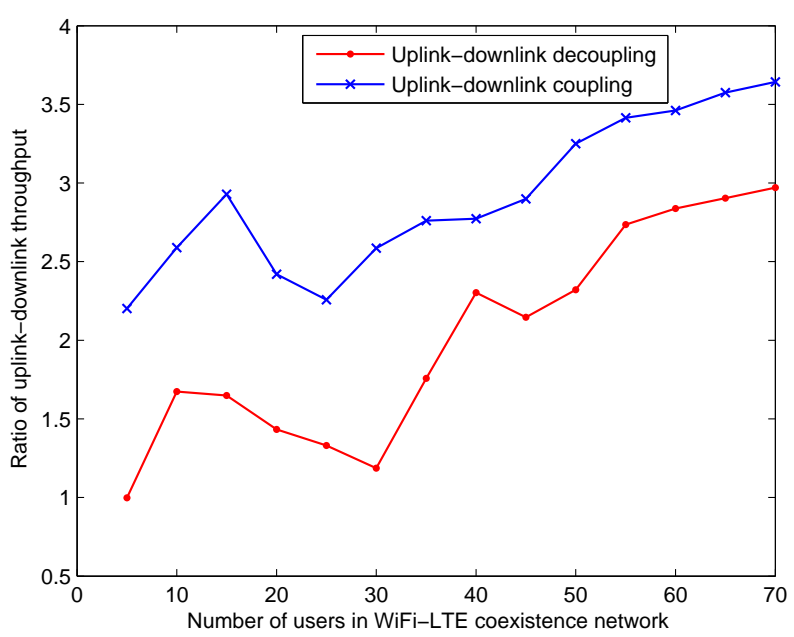

Fig. 8: Ratios of uplink-downlink throughput in WiFi and LTE coexistence network with and without DUDe.

channels will be more unequal. However, DUDe can alleviate the unfairness between uplink and downlink while the total number of network users increases.

Fig. 9 shows the comparison of downlink throughput with its unit of Mbps in WiFi and LTE coexistence network with and without DUDe for 96 hours. We find that the throughput of the downlink with DUDe is larger than that without DUDe at any time. This is because the unfairness of uplink and downlink in WiFi and LTE coexistence network is caused by the WiFi network. And the DCF mechanism in the WiFi network determines that the uplink in the network has more advantages when accessing the channel. Hence, the throughput of the uplink is larger. In order to implement load balance between uplink and downlink, the users will strive for greater downlink throughput after decoupling.

Fig. 10 and Fig. 11 show the comparison of the whole network throughput changing with time and the number of users

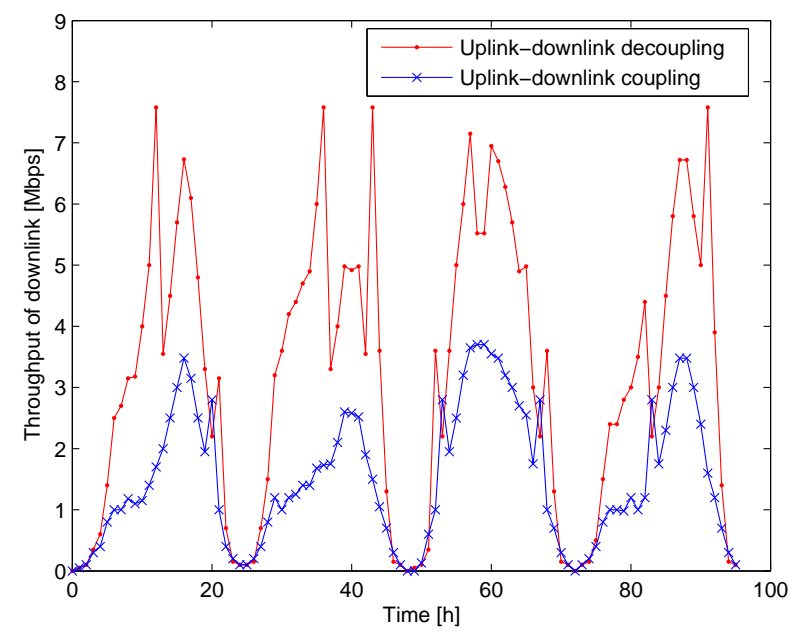

Fig. 9: Downlink throughput in $\mathrm{WiFi}$ and LTE coexistence network with and without DUDe.

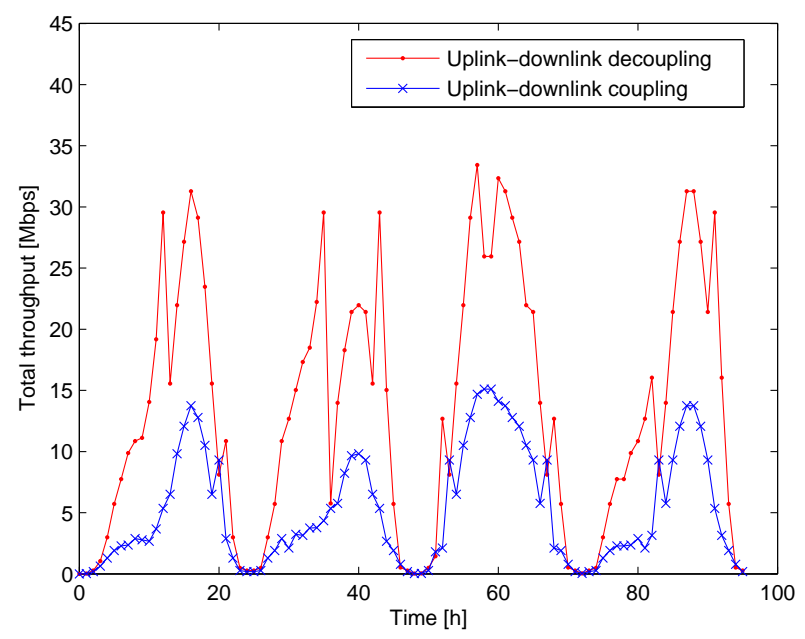

Fig. 10: Total throughput in WiFi and LTE coexistence network with and without DUDe.

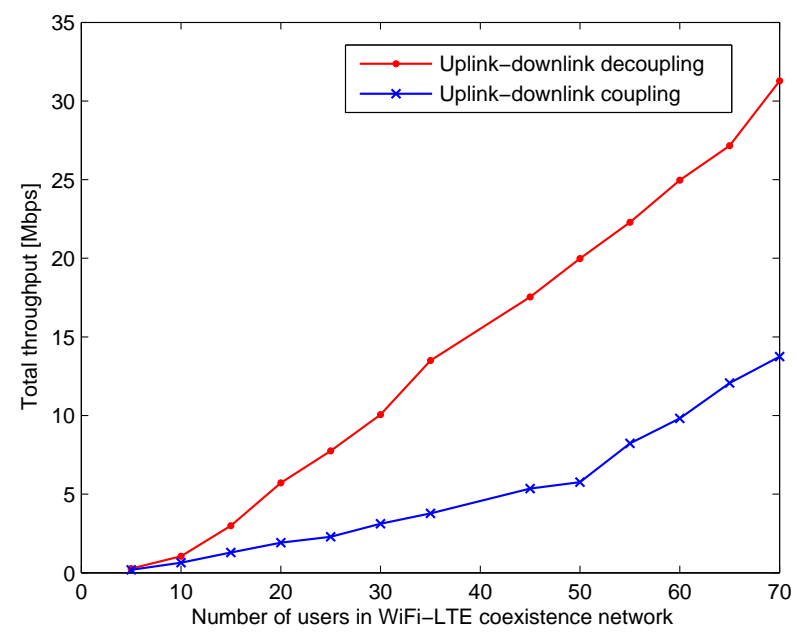

Fig. 11: Total throughput in WiFi and LTE coexistence network with and without DUDe. 
with and without DUDe in WiFi and LTE coexistence network, respectively. From Fig. 10 and Fig. 11, it can be found that, at any time, the total network throughput with DUDe is greater than that without DUDe. And the total network throughput of DUDe scheme can come up to almost twice more than that of coupled association scheme. It indicates that DUDe in WiFi and LTE coexistence network can achieve both load balancing of uplink and downlink and optimize the throughput of coexistence network. As shown in Fig. 11, the throughput of the network increases with the number of users. This is because we can allocate resources in advance according to the total number of users in the future by prediction in the coexistence system. Therefore, the network communication quality can be guaranteed while the number of users increases.

\section{CONClusions}

In this paper, a novel scheme for the WiFi and LTE coexistence is proposed in unlicensed spectrum. In order to achieve load balancing and optimal throughput, we take use of DUDe and combine it with fairness utility function in the coexistence system. Also, performance of our proposed algorithm is compared in the coexistence system with and without DuDe. We find that DUDe can achieve not only load balancing of uplink and downlink but also the optimal throughput of coexistence network. The results shed new light on coexistence between $\mathrm{WiFi}$ and LTE and point out a new direction of achieving fairness between uplink and downlink. Considering the more complex and rich service applications of $5 \mathrm{G}$, our proposed predicted decoupling algorithm would be further updated to meet the coexistence demand between WiFi and $5 \mathrm{G}$. In the future, $\mathrm{WiFi}$ and $5 \mathrm{G}$ may have complementary substitution relationships in specific scenarios, but will still coexist for a long time.

\section{REFERENCES}

[1] Z. M. Fadlullah, et al., "State-of-the-art deep learning: evolving machine intelligence toward tomorrow's intelligent network traffic control systems," IEEE Communications Surveys and Tutorials, vol. 19, no. 4, pp. 2432-2455, Apr. 2017.

[2] M. Liu, J. Yang, and G. Gui, "DSF-NOMA: UAV-assisted emergency communication technology in a Heterogeneous internet of things," IEEE Internet of Things Journal, vol. 6, no. 3, pp. 5508-5519, Jun. 2019.

[3] N. Kato, et al., "The deep learning vision for Heterogeneous network traffic control: proposal, challenges, and future perspective," IEEE Wireless Communications, vol. 24, no. 3, pp. 146-153, Mar. 2017.

[4] F. Tang, et al., "On removing routing protocol from future wireless networks: a real-time deep learning approach for intelligent traffic control," IEEE Wireless Communications, vol. 25, no. 1, pp. 154-160, Jan. 2018.

[5] Y. Zhou, Z. M. Fadlullah, B. Mao, and N. Kato, "A deep-learning-based radio resource assignment technique for $5 \mathrm{G}$ ultra dense networks," IEEE Network, vol. 32, no. 6, pp. 28-34, Jun. 2018.

[6] H. Huang, et al., "Deep Learning for Physical-Layer 5G wireless Techniques: opportunities, challenges and solutions," IEEE Wireless Communications, to be published, doi: 10.1109/MWC.2019.1900027.

[7] F. Tang, et al., "AC-POCA: anti-coordination game based partially overlapping channels assignment in combined UAV and D2D based networks," IEEE Transactions on Vehicular Technology, vol. 67, no. 2, pp. 1672-1683, Feb. 2018.

[8] D. Takaishi, et al., "Virtual cell-based resource allocation for efficient frequency utilization in unmanned aircraft systems," IEEE Transactions on Vehicular Technology, vol. 67, no. 4, pp. 3495-3504, Apr. 2018.

[9] "Enhance mobile networks to deliver 1000 times more capacity by 2020," NSN Whitepapers, Sep. 2013.
[10] “Extending LTE advanced to unlicensed spectrum," Qualcomm Whitepaper, Dec. 2013.

[11] "Qualcomm research LTE in unlicensed spectrum: harmonious coexistence with Wi-Fi," Qualcomm Whitepaper, Jun. 2014.

[12] "Making the best use of unlicensed spectrum," Qualcomm Whitepaper, Sep. 2015 .

[13] “U-LTE: Unlicensed Spectrum Utilization of LTE," Huawei Whitepaper, 2014.

[14] I. Bisio, C. Garibotto, and F. Lavagetto, and A Sciarrone, "Outdoor places of interest recognition using WiFi fingerprints," IEEE Transactions on Vehicular Technology, vol. 68, no. 5, pp. 5076-5086, May 2019.

[15] I. Bisio, C. Garibotto, F. Lavagetto, A. Sciarrone, and S. Zappatore, "Blind detection: advanced techniques for WiFi-based drone surveillance," IEEE Transactions on Vehicular Technology, vol. 68, no. 1, pp. 938-946, Jan. 2019.

[16] I Bisio, C Garibotto, F Lavagetto, A Sciarrone, and S Zappatore, "Unauthorized amateur UAV detection based on WiFi statistical fingerprint analysis," IEEE Communications Magazine, vol. 56, no. 4, pp. 106-111, Apr. 2018.

[17] Y. Jian, C-F. Shih, B. Krishnaswamy, and R. Sivakumar, "Coexistence of Wi-Fi and LAA-LTE: experimental evaluation, analysis and insights," in Proc. IEEE ICCW, pp. 2325-2331, London, UK, Jun. 8-12, 2015.

[18] X. Yuan, X. Qin, F. Tian, Y. T. Hou, W. Lou, S. F. Midkiff, and J. H. Reed, "Coexistence between Wi-Fi and LTE on unlicensed spectrum: a human-centric approach," IEEE Journal on Selected Areas in Coтmunications, vol. 35, no. 4, pp. 964-977, Apr. 2017.

[19] J. Xiao, and J. Zheng, "An Adaptive Channel Access Mechanism for LTE-U and WiFi Coexistence in an Unlicensed Spectrum," in Proc. IEEE ICC, pp. 1-6, Kuala Lumpur, Malaysia, May 23-27, 2016.

[20] C. Chen, R. Ratasuk, and A. Ghosh, "Downlink performance analysis of LTE and WiFi coexistence in unlicensed bands with a simple listenbefore-talk scheme," in Proc. IEEE VTC, pp. 1-5, Glasgow, Scotland, May 11-14, 2015.

[21] Y. Song, K. W. Sung, and Y. Han, "Coexistence of Wi-Fi and cellular with listen-before-talk in unlicensed spectrum," IEEE Communications Letters, vol. 20, no. 1, pp. 161-164, Jan. 2016.

[22] E. Almeida, et al., "Enabling LTE/WiFi coexistence by LTE blank subframe allocation," in Proc. IEEE ICC, pp. 5083-5088, Budapest, Hungary, Jun. 9-13, 2013.

[23] Z. Guan, and T. Melodia, "CU-LTE: Spectrally-efficient and fair coexistence between LTE and Wi-Fi in unlicensed bands," in Proc. IEEE INFOCOM, pp. 1-9, San Francisco, CA, Apr. 10-14, 2016.

[24] H. Boostanimehr, and V. K. Bhargava, "Joint downlink and uplink aware cell association in HetNets with QoS provisioning," IEEE Transactions on Wireless Communications, vol. 14, no. 10, pp. 5388-5401, Oct. 2015.

[25] A. Celik, R. M. Radaydeh, F. S. Al-Qahtani, and M. S. Alouini, "Joint interference management and resource allocation for device-todevice (D2D) communications underlying downlink/uplink decoupled (DUDe) heterogeneous networks," in Proc. IEEE ICC, pp. 1-6, Paris, France, May 21-25, 2017.

[26] F. Boccardi, J. Andrews, and H. Elshaer, "Why to decouple the uplink and downlink in cellular networks and how to do it," IEEE Communications Magazine, vol. 54, no. 3, pp. 110-117, Mar. 2016.

[27] H. Elshaer, F. Boccardi, M. Dohler and R. Irmer, "Downlink and uplink decoupling: a disruptive architectural design for 5G networks," in Proc. IEEE GLOBECOM, pp. 1798-1803, Austinc, USA, Dec. 7-11, 2014.

[28] A. A. Bankole, and S. A. Ajila "Cloud client prediction models for cloud resource provisioning in a multitier web application environment," in Proc. IEEE SOSE, pp. 156-161, Washington, USA, Mar. 25-28, 2013.

[29] G. Bianchi, "Performance analysis of the IEEE 802.11 distributed coordination function," IEEE Journal on Selected Areas in Communications, vol. 18, no. 3, pp. 535-547, Mar. 2000.

[30] J. Mo and J.C. Walrand, "Fair end-to-end window-based congestion control," IEEE/ACM Transactions on Networking, vol. 8, no. 5, pp. 556567 , Oct. 2000

[31] G. Gui, H. Sari, and E. Biglieri, "A new definition of fairness for nonorthogonal multiple access," IEEE Communications Letters, vol. 23, no. 7, pp. 1267-1271, May 2019.

[32] Y. T. Hou, Y. Shi, and H. D. Sherali, "Applied optimization methods for wireless networks," Cambridge University Press, ISBN-13: 9781107018808.2014

[33] C. L. Frenzena, Ts. Sasaob, and J. T. Butlerc, "On the number of segments needed in a piecewise linear approximation," Journal of Computational and Applied Mathematics, vol. 234, no. 2, pp. 437-446, 2010. 
[34] X. Yuan, et al., "Beyond overlay: reaping mutual benefits for primary and secondary networks through node-level cooperation," IEEE Transactions on Mobile Computing, vol. 16, no. 1, pp. 2-15, 2017.

[35] Y. Wang, M. Liu, J. Yang, and G. Gui, "Data-driven deep learning for automatic modulation recognition in cognitive radios," IEEE Transactions on Vehicular Technology, vol. 68, no. 4, pp. 4074-4077, Apr. 2019.

[36] G. Gui, H. Huang, Y. Song, and H. Sari, "Deep learning for an effective nonorthogonal multiple access scheme," IEEE Transactions on Vehicular Technology, vol. 67, no. 9, pp. 8440-8450, Sept. 2018.

[37] J. Wang, et al., "UL-CSI data driven deep learning for predicting DLCSI in cellular FDD systems," IEEE Access, to be published, doi: 10.1109/ACCESS.2019.2929091.

[38] B. Mao, et al., "Routing or computing? The paradigm shift towards intelligent computer network packet transmission based on deep learning," IEEE Transactions on Computers, , vol. 66, no. 11, pp. 1946-1960, Nov. 2017.

[39] J. Sun, et al., "Behavioral modeling and linearization of wideband RF power amplifiers using BiLSTM networks for 5G wireless systems," IEEE Transactions on Vehicular Technology, to be published, doi: 10.1109/TVT.2019.2925562.

[40] H. Huang, et al., "Deep-Learning-based Millimeter-Wave Massive MIMO for Hybrid Precoding," IEEE Transactions on Vehicular Technology, , vol. 68, no. 3, pp. 3027-3032, Mar. 2019.

[41] S. Mukherjee, E. Osuna, and F. Girosi, "Nonlinear prediction of chaotic time series using support vector machine," IEEE Transactions on Neural Networks and Learning Systems, vol. 24, no. 2, pp. 207-218, Jul. 1999.

[42] B. J. Chen, M. W. Chang, and C. J. Lin, "Load forecasting using support vector Machines: a study on EUNITE competition 2001," IEEE Transactions on Power Systems, vol. 19, no. 4, pp. 1821-1830, Nov. 2004.

[43] B. Gui, X. Wei, Q. Shen, J. Qi, and L. Guo, "Financial time series forecasting using support vector machine," in Proc. IEEE CIS, pp. 3943, Kunming, China, Nov. 15-16, 2014.

[44] L. J. Cao, E. Francis, and H. Tay, "Support vector machine with adaptive parameters in financial time series forecasting," IEEE Transactions on Neural Networks vol. 14, no. 6, pp. 1506-1518, Oct. 2003.

[45] V. Cherkassky, "The nature Of statistical learning theory," IEEE Transactions on Neural Networks, vol. 8, no. 6, pp. 1564-1564, Nov. 1997.

[46] F. Liu, et al., "Flight delay prediction based on aviation big data and machine learning," IEEE Transactions on Vehicular Technology, to be published, doi: 10.1109/TVT.2019.2954094.

[47] F. Wen, "Computationally efficient DOA estimation algorithm for MIMO radar with imperfect waveforms," IEEE Communications Letters, vol. 23, no. 6, pp. 1037-1040, Jun. 2019.

[48] F. Wen, Z. Zhang, and X. Zhang, "CRBs for direction-of-departure and direction-of-arrival estimation in collocated MIMO radar in the presence of unknown spatially coloured noise," IET Radar, Sonar \& Navigation, vol. 13 no. 4, pp. 530-537, Apr. 2019.

[49] F. Wen, Z. Zhang, and G. Zhang, "Joint DOD and DOA estimation for bistatic MIMO radar: A covariance trilinear decomposition perspective," IEEE Access, vol. 7, no. 1, pp. 53273-53283, 2019.

[50] X. P. Wang, et al., "Nuclear norm minimization framework for DOA estimation in MIMO radar," Signal Processing, vol. 135, pp. 147-152, June 2017.

[51] H. Wang, et al., "Assistant vehicle localization based on three collaborative base stations via SBL-based robust DOA estimation," IEEE Internet of Things Journal, vol. 6, no. 3, pp. 5766-5777, June 2019.

[52] B. Wang, et al., "Spatial- and frequency-wideband effects in millimeterwave massive MIMO systems," IEEE Transactions on Signal Processing, vol. 66, no. 13, pp. 3393-3406, July 2018.

[53] H. Xie, F. Gao, S. Zhang, and S. Jin, "A unified transmission strategy for TDD/FDD massive MIMO systems with spatial basis expansion model," IEEE Transactions on Vehicular Technology, vol. 66, no. 4, pp. 3170 3184, Apr. 2017.

[54] I. Bisio, F. Lavagetto, M. Marchese, and A. Sciarrone, "Comparison of Situation Awareness Algorithms for Remote Health Monitoring with Smartphones," in Proc. IEEE GLOBECOM, pp. 2454-2459, Austinc, USA, Dec. 7-11, 2014.

[55] A. Fleury, M. Vacher, and N. Noury, "SVM-based multimodal classification of activities of daily living in health smart homes: sensors, algorithms, and first experimental results," IEEE Transactions on Information Technology in Biomedicine, vol. 14, no. 2, pp. 274-283, Mar. 2010. 